\title{
Inflatably Deployed Membrane Waveguide Array Antenna for Space
}

\author{
David Lichodziejewski* \\ L'Garde, Inc. \\ Dr. Robin Cravey ${ }^{\dagger}$ \\ NASA LaRC \\ Glenn Hopkins \\ Georgia Technical Research Institute
}

\begin{abstract}
$\underline{\text { Abstract }}$
As an alternative to parabolic antennas and Synthetic Aperture Radar (SAR) systems, waveguide arrays offer another method of providing $\mathrm{RF}$ transmit/receive communication apertures for spacecraft. The advantage of the membrane waveguide array concept, in addition to its lightweight and low packaged volume, is its inherent shape. Relative to parabolic antennas, the requirement to make an accurate doubly curved surface is removed. L'Garde and Langley Research Center (LaRC), are currently working in this area to develop lightweight waveguide array technologies utilizing thin film membrane structures. Coupled with an ultralightweight inflatably deployed rigidizable planar support structure, the system offers a very compelling technology in the fields of space-based radar, communications, and earth resource mapping.
\end{abstract}

\section{Introduction}

After significant development, and several prototypes, a packagable $2.2 \mathrm{~m} \times 1.1 \mathrm{~m} \quad \mathrm{RF}$ prototype was constructed and sent to the Langley Electromagnetics Branch and the Georgia Technical Research Institute for RF testing Figure 1. The membrane waveguides are visible as the large planar array in the center of the structure. Also visible are the radiating slots cut into the upper membrane to transport the RF energy in and out of the waveguide cells. The structure surrounding the waveguide is constructed of rigid aluminum tubes and is designed to simulate the geometry of a deployable rigidizable structure. The innovative RF distribution network is visible on the far end

*leo@lgarde.com, tr.l.cravey@larc.nasa.gov, łglenn.hopkins@gtri.gatech.edu, “Copyright 2002 by L'Garde Inc. Published by the American Institute of Aeronautics and Astronautics, Inc. with permission" of the array, and distributes the RF signal from a single source to each of the eight waveguide feeds. Work is currently ongoing to develop a rigidizable inflatably deployed structure to deploy and support the waveguide array thus completing the antenna system.

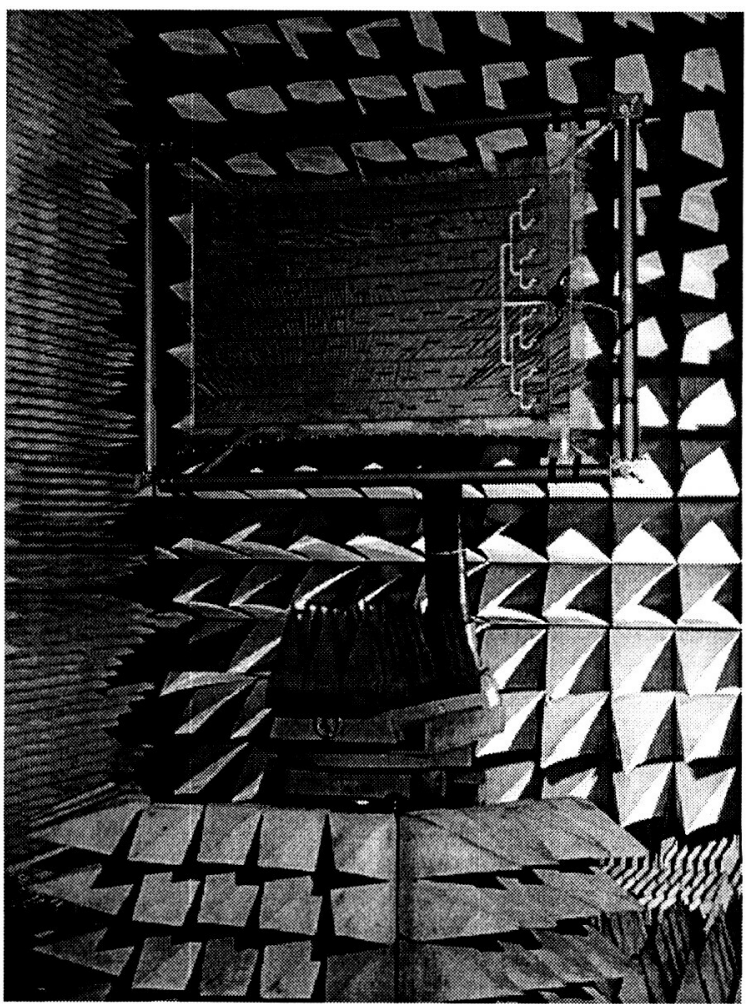

Figure 1. Waveguide Array in RF Test Chamber

This paper discusses the mechanical and structural aspects of the waveguide array and support structure. Excellent RF performance was achieved by the test article, Figure 1, during a comprehensive RF test program at LaRC and GTRI utilizing far and near-field RF testing. The reader is urged to review reference 1 for a detailed description of the test methods and RF performance results. 


\section{System Design}

The design of the thin film waveguide is based on a series of waveguide elements or cells which are bonded together to form a large array. Each waveguide element is a rectangular-shaped prismatic closed shell fabricated from a $0.01 \mathrm{~mm}$ thick metalized Kapton membrane. Each waveguide cell features a feed assembly located near one end. The waveguide array, shown in Figure 2, is made up of eight waveguide cells; the cell cross-sections are just visible at the foreground end of the array toward the right. Slots etched into the metalization are clearly visible and direct RF energy incident on the array down the cell onto the feed assembly just visible toward the background end of the array toward the left. Two catenary sheets supported at the by a rigid simulated inflatable rigidizable structure form the top and bottom membrane surfaces of the waveguide array cells. The catenaries are supported at the corners by cables that transfer the tension loads in the array to the support structure.

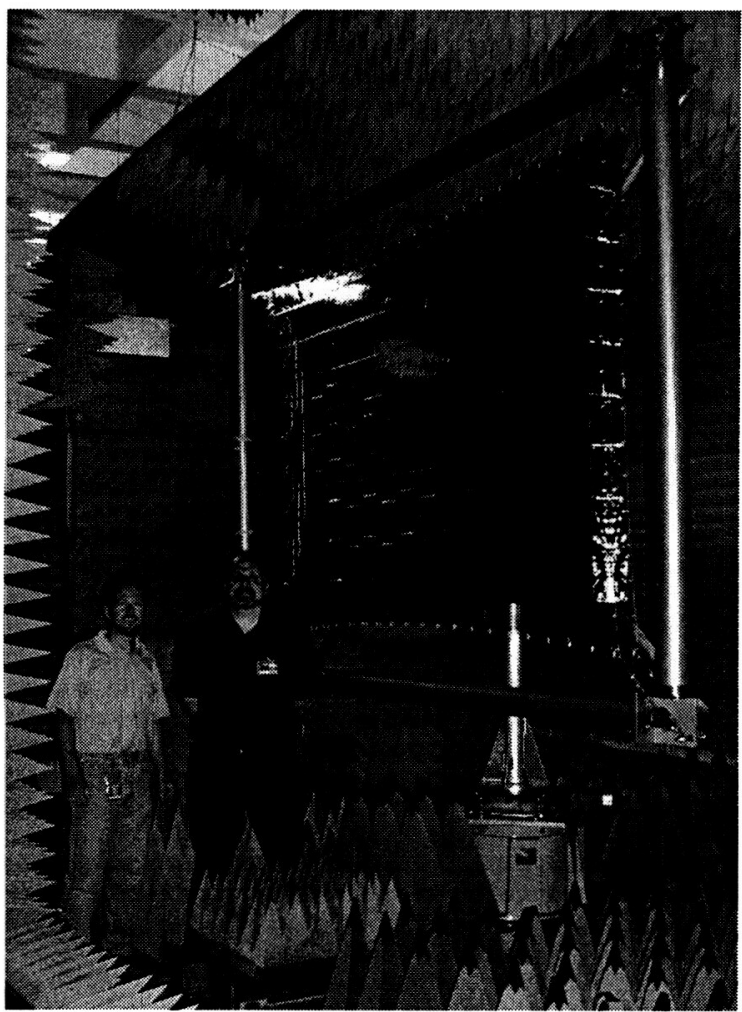

Figure 2. Waveguide Array in GTRI RF Test

Between the adjacent walls of each cell is a $0.13 \mathrm{~mm}$ thick Kapton "stiffener." These stiffeners provide the additional rigidity needed in the vertical direction along each of the waveguide cell walls. These stiffened vertical walls of each cell are perpendicular to the top and bottom surfaces of the array when tensioned by the catenaries.

\section{Baseline Mission}

Much interest has been shown in this concept as a water sensing radiometry antenna for Earth resource mapping. A highly enhanced inflatably deployed rigidizable support structure has been developed around the baseline $9 \mathrm{~m} \times 9 \mathrm{~m}$ radiometer mission. To make up the array, for the $9 \mathrm{mx} 9 \mathrm{~m}$ unit, 55 waveguide elements will be bonded together. A structural optimization resulted in the geometry shown. The tube geometries were selected to withstand the tension loads in the array with minimum safety factors of 2.0 in local buckling, and 4.0 in Euler buckling, while minimizing the mass of the overall structure.

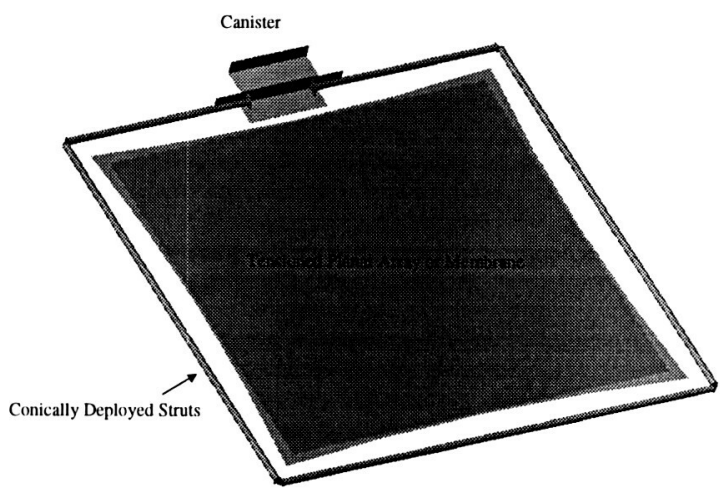

Figure 3. Optimized $9 m^{*} 9 m$ Radiometer Array

\section{Feed Network}

To transport the RF signal from the transceiver to the waveguide cells a packagable integrated feed and microstrip network has been designed and fabricated. The design, developed by LaRC and GTRI and fabricated by L'Garde, utilizes metalized membranes supported by a low dielectric foam material to provide the proper impedance for efficient RF transport. The network is mounted above the waveguide array, which concurrently acts as an RF groundplane for the network. Feeds are integrated into the network, which transport the signal to and from the waveguide cell. The network is designed to divide/combine the signal through 8 separate and equal length paths. A picture of the network mounted to the waveguide array is 
shown in Figure 4, note the feed elements extend through circular holes in the cell walls.

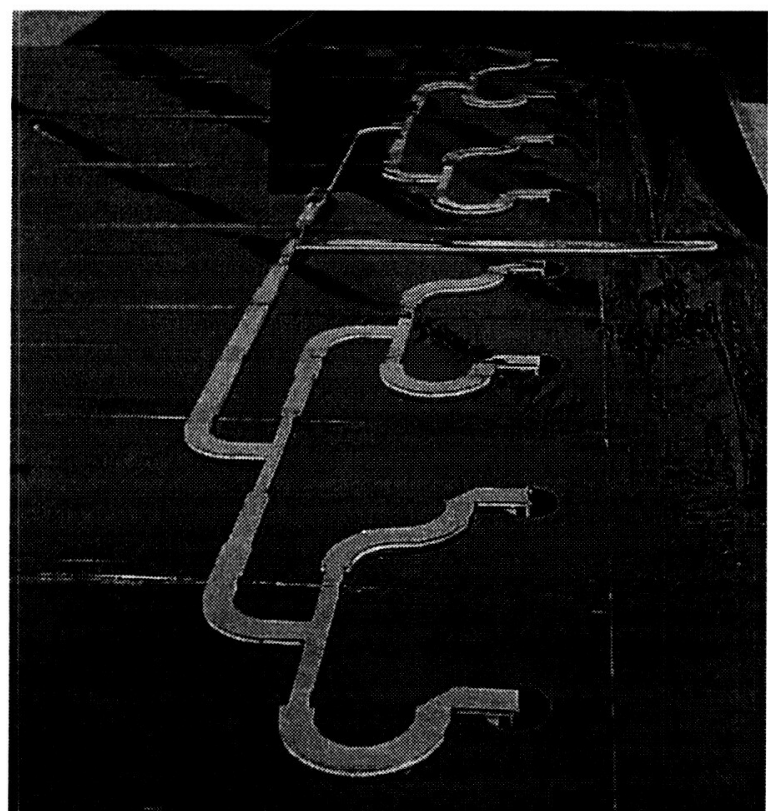

Figure 4. 8:1 Combiner Microstrip Network

Novel "hinges" in the form of precisely positioned gaps in the foam were integrated into the network design and fabrication. These "hinges" allow the network to be folded and are positioned to align with the array fold lines for packaging. Precise geometries were attained by using a computer-controlled laser cutting table to form the foam and membrane structure.

\section{Array Stowage}

The waveguide cells are stowed by folding the top and bottom membranes between the sidewall stiffeners as shown in Figure 5. Once the cells are folded in this manner, the sidewall stiffeners are brought together in an accordion fashion.

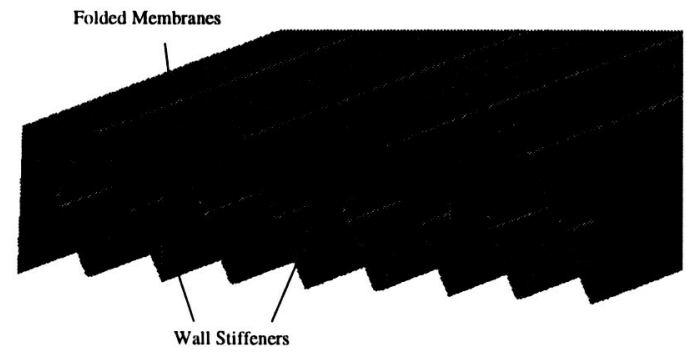

Figure 5. Waveguide Cell Packaging Concept

The thicker Kapton sidewall material resists folding and must be rolled in a radius to avoid permanently deforming the material. The resulting serpentine packaging concept for the stowed waveguide is visible in Figure 6 . The serpentine waveguides are looped back resulting in a very efficient packaging arrangement. The feeds and microstrip network fully stowed toward the foreground.

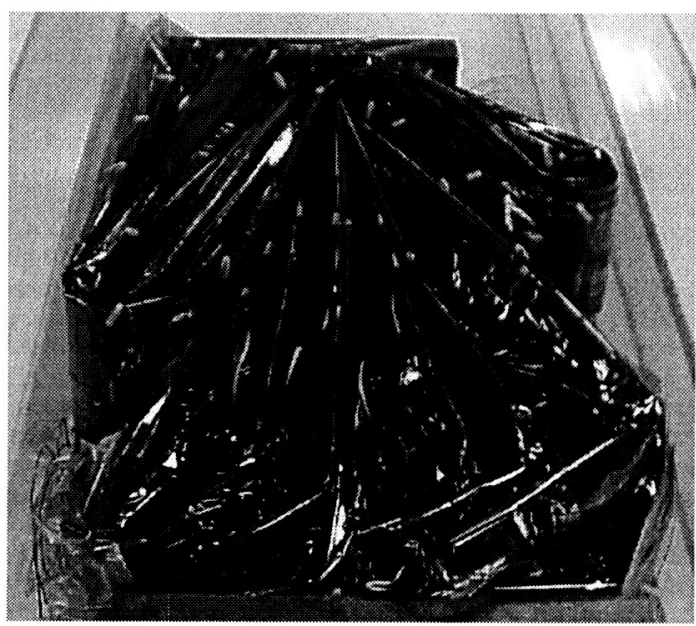

Figure 6. Packaged Waveguide Array

\section{Sub-Tg Rigidization}

Sub-Tg or Cold rigidization takes advantage of the increase in modulus of certain materials below their glass transition temperature $(\mathrm{Tg})$. The system is made up of a specialized composite weave utilizing strong, lightweight fibers impregnated with a L'Garde formulated sub-Tg elastomer. Though sometimes called cold rigidizables they don't necessarily require cold temperatures. Elastomers can be formulated which rigidize at temperatures tailored to specific mission requirements. Cold rigidized structures can be constructed for a variety of missions, from LEO to deep space applications and this technique was selected to form the support structure for the waveguide array

A lightweight sub-Tg rigidizable truss built by L'Garde is shown in Figure 7. This structure has been mechanically tested at LaRC and withstood 2473.21N. in compression, though its own mass does not exceed $4.31 \mathrm{~kg}$. This particular truss uses a Sub-Tg resin formulated to be rigid at room temperature, with a $\mathrm{Tg}$ (glass transition temperature) of about $+40 \mathrm{C}$, this very large structure can be tested at room temperature conditions. 


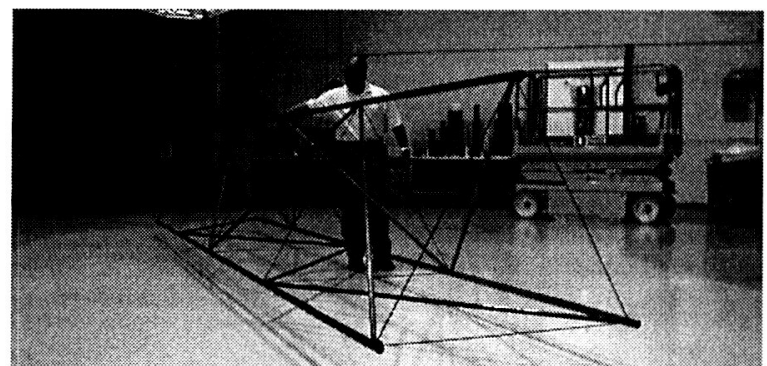

Figure 7. Sub-Tg 8m Rigidizable Space Truss

Sub $\mathrm{Tg}$ rigidizable structures are simple and reliable. They are completely passive and in general require no heaters or vents. However, since their rigidization depends on temperatures below their $\mathrm{Tg}$, a thorough understanding of the thermal environment is required. Ideally, an elastomer is selected with a $\mathrm{Tg}$ below the equilibrium temperature of the spacecraft during deployment. After inflation and deployment, outside of the spacecraft's thermal environment, the structure cools and becomes rigid. If the deployed structure must endure large thermal excursions, it will be tailored to have a higher transition temperature and heaters may be required to "soften" the structure for deployment. MLI is required to mitigate the effects of on-orbit thermal gradients and to retain the initial softening thermal energy during the deployment sequence.

\section{Conical Deployment}

Figure 8 shows the conical boom packaging and deployment scheme developed for deployment control of the inflatable rigidizable support booms. The technique uses a unique concentric packaging arrangement about the boom axis and provides a high degree of deployment control (patent pending). In this technique, a tapered tube is packaged in a manner similar to a telescoping tube. Because there is essentially zero excess mass associated with the deployment control method, it scales very well and provides excellent packaging efficiency. To deploy the conical boom, inflation gas is introduced at the base. Pressure is exerted against the walls of the tube, the tip, and base endcaps. The inflation pressure unfolds and deploys the leading folds. The resulting deployment is very smooth and predictable. As the tube is under pressure during deployment, it is able to withstand some load while deploying.

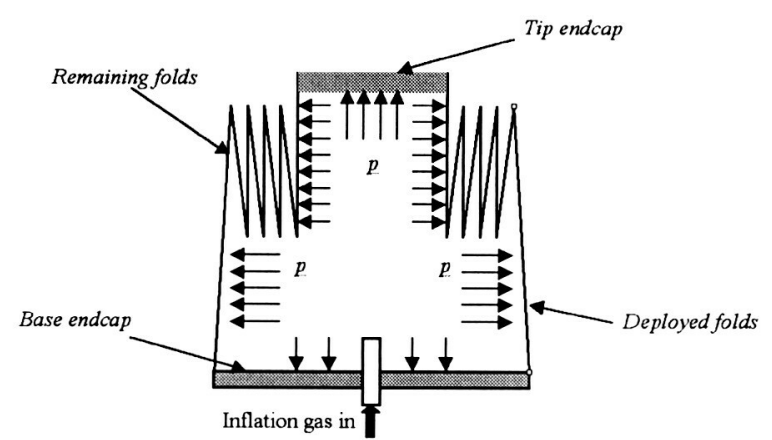

Figure 8. Conical Inflation Schematic

A deployment sequence of a conical boom is shown in Figure 9. The deployment proceeded smoothly and in a linear, consistent, and predictable manner. This particular tube is fabricated of a specialized Kevlar weave impregnated with L'Garde's L6 -20C sub Tg resin. The tube is $20.32 \mathrm{~cm}$ in diameter and is over $2.54 \mathrm{~m}$ long. Similar tubes have been mechanically tested under simulated space thermal conditions. The tubes achieved a material modulus of $26.89 \mathrm{gpa}$ after enduring conical packaging and deployment.
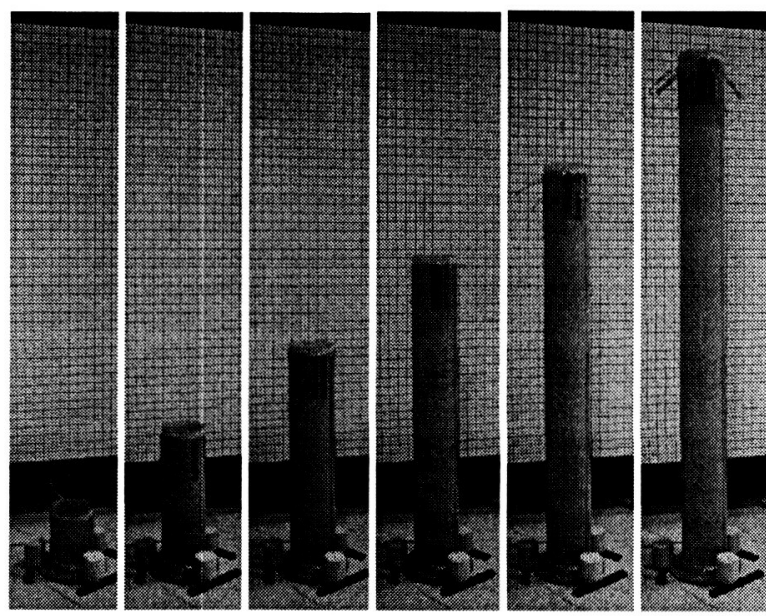

Figure 9. Conical Deployment Sequence

\section{Stowed Configuration}

A stowage and deployment concept has been developed around the $9 \mathrm{mx} 9 \mathrm{~m}$ baseline mission. The concept marries the stowage capabilities of the waveguide array and the unique highly controlled deployment of the conically deployed sub-Tg rigidizable struts. The stowed configuration is shown in Figure 10. The conically packaged struts are shown stowed around the outside of the serpentine folded array. Since some clearance is required between the stowed tubes to encompass the stowed 
array, a small portion of the front lateral tube seen in the foreground is partially deployed and flattened to connect the corner braces. The volume at the base of the canister contains the inflation tanks and inflation/deployment system.

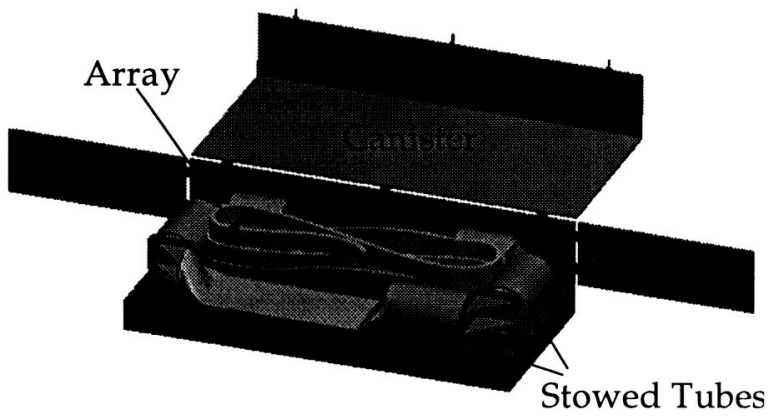

Figure 10. Stowed Waveguide Configuration

\section{Deployment}

A schematic of the deployment sequence of the $9 \mathrm{mx} 9 \mathrm{~m}$ baseline design utilizing conically deployed booms is shown in Figure 10. Upon initiation of the deployment sequence, the top and sides of the canister are opened to release the array and support structure. The deployment in the longitudinal direction is initiated first to extend the stowed waveguide array. Two opposed double conically deployed struts are used to control the sequence in the longitudinal direction. Conical struts on either side of the canister $(1,2)$ are deployed in parallel. Once these longitudinal struts are at full extension, the waveguide sidewalls are also fully extended. Three conically deployed struts (two single and one double) are used to control the lateral deployment; struts $(3,4)$ are extended and coordinated with the far lateral strut (5). Once all the struts are fully extended and rigidized, the waveguide array is fully deployed.

Once the structure cools to space steady state temperatures rigidization is complete and the inflatant can be vented, as it is no longer required for structural stiffness. The coordination of the conical deployment and inflation will be computer controlled, and techniques for monitoring the deployment

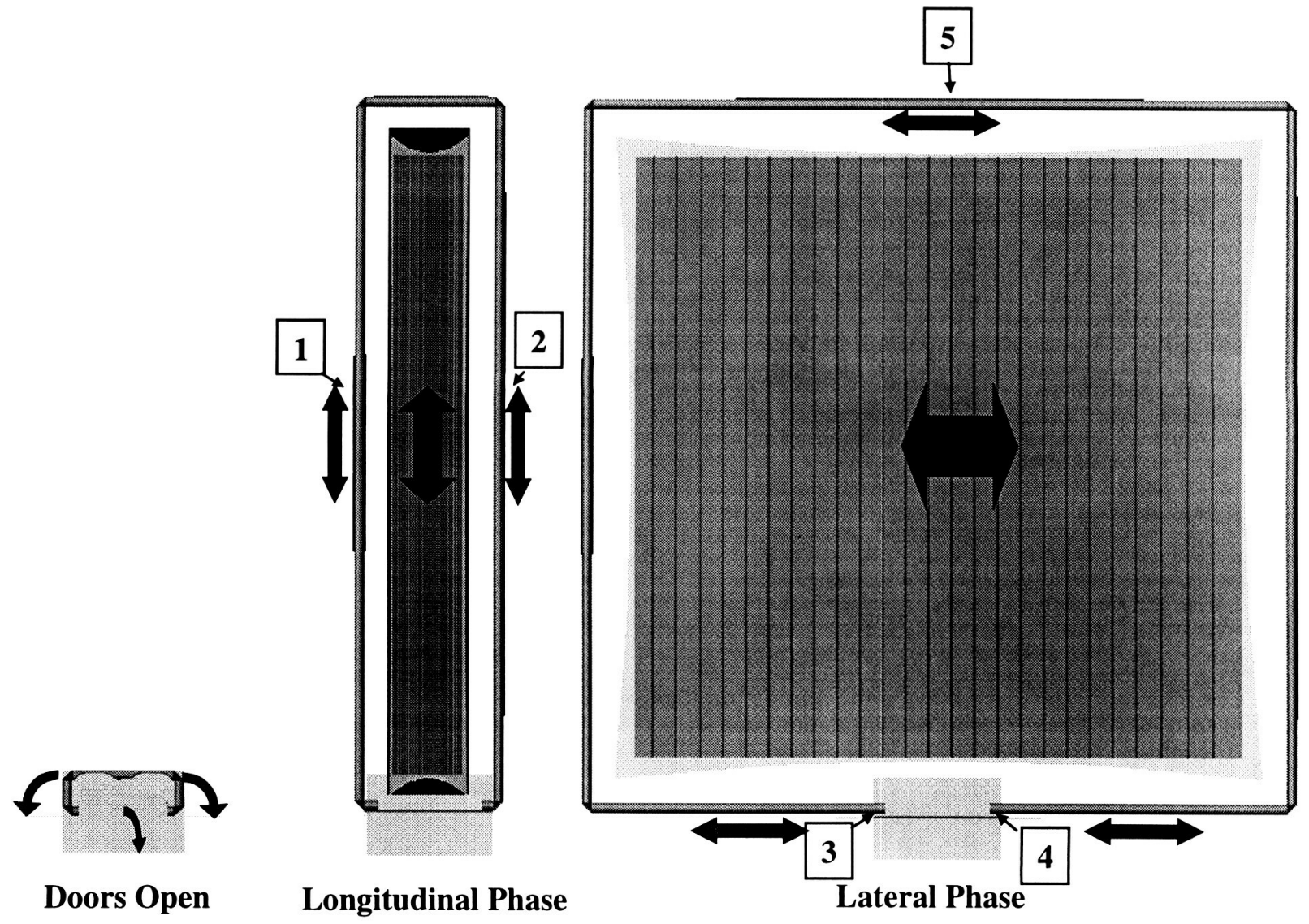

Figure 10. Waveguide Deployment Sequence 
extension of the booms are in review.

\section{Mass/Volume Estimates}

Preliminary mass and volume estimates have been generated for the updated $9 \mathrm{~m} \times 9 \mathrm{~m}$ design. These estimates are based on analytic predictions of prototype waveguide units, measurements of component hardware, and L'Garde experience in other space inflatable programs. The total subsystem mass for the inflatable waveguide is $56.7 \mathrm{~kg}$, yielding an aperture areal density of $0.7 \mathrm{~kg} . / \mathrm{m}^{2}$. The stowed volume of the canister is $0.57 \mathrm{~m}^{3}$.

A comparison of the predicted $9 \mathrm{mx} 9 \mathrm{~m}$ waveguide subsystem mass with a similarly sized rigid deployable antenna is shown in Figure 11. The rigid system masses are for Astro Aerospace's AstroMesh parabolic deployable antennas, see reference 2 . The inflatable waveguide system mass compares favorably to the rigid parabolic reflector masses. Note, however, other factors are introduced when comparing parabolic and planar antennas. Parabolic antennas can function well over a large frequency range while the waveguide is optimized for a small frequency range. The waveguide, however, can be electronically beam steered, by phasing the waveguide feeds, decreasing pointing requirements, and increasing mission applicability. This comparison is meant only to show competitive system masses with other technologies.

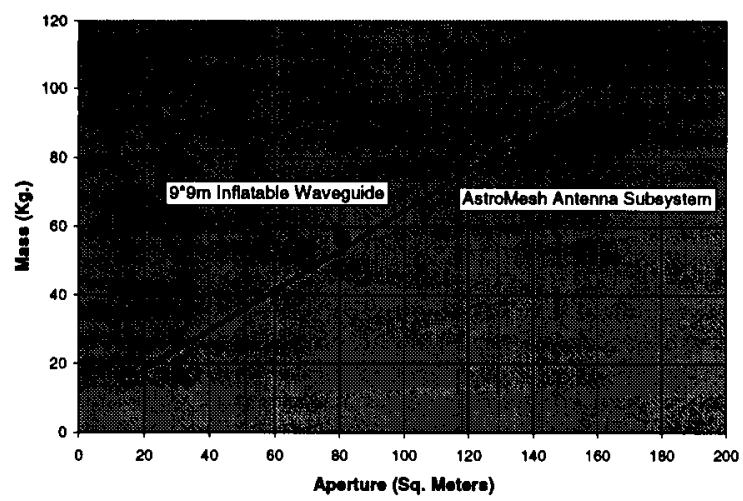

Figure 11. Waveguide Mass Comparisons

\section{Conclusions}

In this series of programs, L'Garde, LaRC, and GTRI have continued development of the membrane Waveguide Array concept and the deployable/rigidizable tensioned membrane support structure. Several prototypes have been fabricated and a fully functional and packagable membrane waveguide array has been fabricated and demonstrated. The array features a novel packagable feed and signal distribution network. RF testing at LaRC and GTRI has shown the feasibility and performance of the design. A support structure featuring sub-Tg rigidizable conically deployed booms has been designed and test articles of these booms have been fabricated, deployed, and mechanically tested under cold conditions to validate the deployment and mechanical properties. A NASA SBIR Phase II effort is currently underway which will further develop the waveguide array support system, culminating in thermal vacuum deployment and rigidization of the waveguide array and support structure under simulated space conditions. The commercial applications of the membrane planar waveguide concept are numerous. The lightweight, low stowed volume, and simplicity of the concept, coupled with the geometric precision of the planar array, will find many applications in the fields of space-based radar, communications, and earth resource mapping.

\section{Acknowledgments}

Much appreciation is extended to Tom Campbell and M.C. Bailey, formally of NASA LaRC, for their vision, advocacy, and technical expertise in development of the membrane waveguide array. The authors would also like to thank Wes Lawrence of NASA LaRC Electromagnetics Branch for his continued support and expertise. L'Garde would like to thank Dr. Gyula Greschik for his assistance in the waveguide array and deployable support structure design and analysis.

\section{$\underline{\text { References }}$}

1) R.L. Cravey, D. Lichodziejewski, G. Hopkins, D. Fralick, M. C. Bailey, "Performance Evaluation of a Membrane Waveguide Array Antenna", IEEE-20031148 IEEE Aerospace Conference, March 8 2003, WY

2) Brandt, D., "Thin Film Waveguide Conceptual Design Study", L'Garde Technical Report, LTR-98-DFB-003 
3) Thompson, M., "The AstroMesh Deployable Reflector", Proceeding of fifth International Satellite Conference , June 1997

4) Guidanean, K, Lichodziejewski, D. "An Inflatable Rigidizable Truss Structure Based on New Sub- Tg Polyurethane Composites", AIAA-2002-1593, 43 ${ }^{\text {rd }}$ AIAA Aerospace Sciences Meeting and Exhibit, Apr. 25, 2002, Denver, $\mathrm{CO}$

5) R.L. Cravey, H.-J.C. Blume, C.P. Hearn, M.J. Scherner, L.C. Schroeder, and R.F. Harrington, "Electromagnetic Losses in Metallized Thin Films for Inflatable Radiometer Applications," Proceedings of the AIAA 1995 Space Programs and Technologies Conference, September 26-28, 1995, Huntsville, AL, AIAA Paper 95-3741.

6) M.C. Bailey and T. G. Campbell.: "Inflatable Tensioned Membrane Waveguide Antenna Array for Space Applications", IUTAM-IASS Symposium on Deployable Structures, Cambridge, UK, September 6-9, 1998.

7) G.D. Hopkins, "Near-Field Measurement and Evaluation of Tensioned Membrane Array Antenna", NASA LaRC NAS1-02056, Task Order 6003, August 10, 2002 